\title{
sciendo
}

10.2478/AMB-2020-0022

\section{CHYLOTHORAX AFTER CARDIAC SURGERY}

\author{
Z. Vazhev, K. Dimitrov, H. Stoev \\ Department of Cardiac and Vascular Surgery \\ Medical University - Plovdiv, Bulgaria
}

\begin{abstract}
In the following article we present a case of postoperative chylothorax as rare and severe complication after cardiac surgery. We present a case of 56-year-old man after double-valve replacement procedure and aorto-coronary artery bypass grafting, including pedicled left internal mammary artery towards left anterior descending artery. By the tenth postoperative day the total amount of drained milky white fluid from the left pleural cavity reached the colossal volume of $13040 \mathrm{ml}$, despite the conservative therapy with intravenous Sandostatin (Octreotide) and parenteral feeding with a solution rich in medium chain triglycerides and amino acids - OliClínomel. The results of the biochemical analysis confirmed the diagnosis chylothorax. On the tenth postoperative day, a revision of the left pleural cavity through left thoracotomy was performed and lesion of the thoracic duct was identified. The lesion was sutured and secured with fibrin tissue glue - Tissucol for definitive treatment of the lymphorrhagia. The combined therapeutic and surgical approach concerning this serious complication turned out to be effective, and the patient was discharged on the thirty-second postoperative day with significant clinical improvement without ultrasound and $x$-ray data for left pleural effusion.
\end{abstract}

Key words: chylothorax, double-valve replacement, left internal mammary artery, complications

Corresponding author: Assistant-professor Hristo Angelov Stoev, Department of Cardiac and Vascular Surgery, Medical University, Sveti Geogi University Hospital Plovdiv, Bulgaria, e-mail: hristostoev87@gmail.com

\section{INTRODUCTION}

$\longrightarrow$ hylothorax is an abnormal collection of lymph fluid in the pleural cavity that most commonly occurs as a result of a lesion of the thoracic duct (ductus thoracicus) or its branches. The lymphatic pleural effusion is rare but serious complication after cardiac surgery. The symptoms of chylothorax

are no different from the other clinical conditions, associated with pleural effusion, which are confirmed by anamnestic, physical, clinical and imaging-diagnostic methods: ultrasound, chest x-ray, CT with contrast enhacement, MRI. The chylothorax has typi- cal biochemical characteristics from the pleural fluid analysis: elevated levels of triglycerides (their level in the lymph fluid is two to eight times higher than in the plasma), low levels of cholesterol (its level is lower than that in plasma), lower values of total protein and albumin, elevated lymphocyte levels, presence of erythrocytes, chylomicrons and electrolytes. The macroscopic characteristic of the chylose fluid is specific - white, milklike, odorless. The clinical manifestation of chylothorax changes with time. In the early stages of its development there are no typical symptoms, but in the late stages the dyspnea becomes the main symptom, when great amount of 
lymph fluid is collected in the pleural cavity. In the advanced stages of chylothorax the clinical signs of malnutrition caused by loss of proteins, lipids, immunoglobulins, electrolytes and water are manifested.

The following etiological forms of chylothorax are distinguished:

1. Congenital chylothorax: With very rare incidence, it is observed during the neonatal period and is associated with birth trauma and/or complication from anatomical anomalies [1].

2. Traumatic chylothorax: The mechanism of the trauma of the thoracic duct following blunt trauma include extension of the spine with rupture of the thoracic duct in the diaphragm. Penetrating traumas and damages of the thoracic duct are extremely rare. Typical for penetrating traumas of the thoracic duct is that when the lesion is above the level of Th- 5 , it is accompanied by the development of left-sided chylothorax, and when it is below Th-5, it is accompanied by a right-sided chylothorax, respectively [2].

3. Chylothorax in infectious diseases: Exclusively rare cases like tuberculous lymphadenitis, filariasis or ascending lymphangitis may be complicated by chylothorax resulting from thoracic duct obstruction.

4. Chylothorax in malignant and benign neoplasms: Obstruction of the thoracic duct is due to thoracic neoplasia (most often seen with lymphoma), which can lead to overstreching and tearing of its wall. Sometimes it is also observed in direct invasion of epithelial tumor (lung or esophageal carcinoma). Chylothorax, although very rarely, can also be accompanied by a benign tumor (lymphangioma, mediastinal cystic hygroma).

5. Chylothorax in other pathological conditions: Spontaneous chylothorax due to severe cough or vomiting accompanied by diaphragm laceration; chylothorax in sarcoidosis; chylothorax in cirrhosis; chylothorax in subcalvian vein or superior vena cava thrombosis; chylothorax in lymphagiomyomatosis; idiopathic chylothorax.

6. Postsurgical chylothorax and/or iatrogenic chylothorax: Often observed in surgical interventions on the esophagus, larynx, lungs, mediastinum and heart. As a complication, chylothorax can be observed after cannulation of the large arteries and veins. Chylothorax can also be seen after radiation therapy.

In cardiac surgery the development of postoperative chylothorax is rarely encountered complication. Kilic and collaborators have observed this complication in less than $0.1 \%$ of patients who underwent surgery for myocardial revascularization. Postoperatively it can develop after procedures in the region of the anterior mediastinum due to a damage of the aberrant lymphatic vessels and thymus or laceration of the thoracic duct during preparation and manipulation on the outgoing from the heart arterial vessels for correction like aortic aneurysm repair, persistent ductus arteriosus (PDA), coarctation of the aorta (CoA). Chylothorax after coronary artery bypass surgery is due to damage to the lymphatic pathways during the harvesting of the left internal thoracic artery. During the preparation of the mammary artery, the thoracic duct could be affected in the region of the venous confluence of the jugular and subclavian vein or collateral lymphatic vessels could be damaged proximal to the entering of the thoracic duct into the venous system.

\section{CASE REPORT}

A 56-year-old man indicated for elective surgical intervention was admitted to the hospital. The surgical procedure included mitral valve and aortic valve replacement surgery with mechanical mitral prosthesis and myocardial revascularization of the left anterior descending artery (LAD) using the left internal thoracic artery as arterial graft. The patient had a history for rheumatism in childhood with diagnosed "heart murmur". Percutaneous coronary intervention was performed 1 year prior to the surgical intervention and bare-metal stent was implanted in the proximal segment of the left anterior descending artery. The patient had complaints of dyspnoea, fatigue and precordial pain. After $\mathrm{PCl}$ and heart team discussion, the patient was indicated for cardiac surgical intervention. The patient also had diabetes for about 15 years, atrial fibrillation for 5 years and was hepatitis $C$ positive.

Pre-operative echocardiographic study demonstrated a normal left ventricle ejection fraction of $57 \%$ by Teicholz and fractional shortening of $32 \%$. The echocardiographic diameter of the aortic valve ring was $21 \mathrm{~mm}$. Aortic bulb-35 mm, ascending aorta-34 $\mathrm{mm}$. The native aortic valve was tricuspid with fibrodegenerative changes and moderate to severe aortic valve stenosis with a peak systolic transvalvular gradient of $67 \mathrm{~mm} \mathrm{Hg}$, and 1st degree of aortic valve regurgitation.

Echocardiography found moderate to severe left ventricular hypertrophy: interventricular septum with a thickness of $16 \mathrm{~mm}$, posterior wall of the left ventricle - $15 \mathrm{~mm}$. The following measurements were within the normal range: EDD - $42 \mathrm{~mm}$, when the normal M-value mode is $35-55 \mathrm{~mm}$; ESD - $29 \mathrm{~mm}$, at normal 
25-38 mm; EDV of $89 \mathrm{ml}$ (normal range is less than $150 \mathrm{ml}$ ), ESV $39 \mathrm{ml}$ (normal range is less than 50 $\mathrm{ml}$ ). Dilated left atrium measured by two-dimensional echography - 5.1-6.4 mm, from an apical position and area of the left atrium of $30 \mathrm{~cm}^{2}$. Transthoracic echocardiographic study demonstrated severe mitral valve stenosis with a mean gradient of $17 \mathrm{mmHg}$ and mitral valve annulus (MVA) $-0.83 \mathrm{~cm}^{2}$.

Doppler-echocardiography study demonstrated decreased mitral blood flow: E-wave-2.77 m/s. and Awave-2.49 m/s.; E/A-1.11; PHT (pressure half time) - $264 \mathrm{~m} / \mathrm{s}$. Echocardiographically, dilated right ventricular cavities were established - right ventricle EDD of $30 \mathrm{~mm}$ and right atrium size of $4.6-5.1 \mathrm{~mm}$. Preoperative $\mathrm{PCl}$ demonstrated right dominant coronary artery circulation. Two-vessel coronary artery disease was found with non-significant stenosis of the left main of LCA. Selective coronary angiography demonstrated in-stent restenosis in the proximal LAD, absence of plaques in LCx and non-specific lesions in the middle and distal segment of RCA.

Aortography was negative for aortic valve regurgitation, but ventriculography was not performed for positioning difficulties of the catheter through the stenotic valve in the outflow tract of the left ventricle. The patient was tested for the in-stent restenosis in the proximal segment of LAD with intravascular ultrasound. Significant in-stent restenosis up to $3.5 \mathrm{~mm}$ in the distal segment of the implanted stent and nonspecific lesion of the distal region of the left main up to $8 \mathrm{~mm}$.

In conclusion, the preoperative echocardiographic study confirmed the presence of severe aortic and mitral valve stenosis and selective coronary angiography - presence of in-stent restenosis in the proximal segment of LAD.

\section{SURGICAL TECHNIQUE}

Through a median sternotomy, under the terms of cardiopulmonary bypass, moderate hypothermia $-32{ }^{\circ} \mathrm{C}$ and cardioplegic arrest with pedantic myocardial protection management with the use of cold blood cardioplegia introduced in a retrograde fashion through the coronary sinus and antegrade through the coronary artery ostia. The aortic valve was tricuspid with severe degeneration and calcification of the aortic valve leaflets and ring. The aortic valves leaflets were excised and debridement of the calcium deposits in the ring was performed. After standard left atriotomy in the Sondergaard groove, the mitral valve apparatus was exposed. Severe adhesions in the anterolateral and posteromedial commissure were detected, as well as calcium deposits on the mitral valve ring. The two mitral valve leaflets were excised and debridement of calcium deposits on the mitral valve ring was performed. Mechanical bileaflet mitral valve prosthesis "Sorin Carbomedics Standard Mitral Valve \# 27 mm. (Sorin Group, Milan, Italy) was implanted with 12 single U-shaped 2/0 TiCron (Covidien) sutures located at the atrial surface of the mitral valve ring. Mechanical bileaflet aortic valve prosthesis "Sorin Carbomedics Standard Aortic Valve \# 27 mm (Sorin Group, Milan, Italy) was implanted using 15 single U-shaped 2/0 TiCron (Covidien) sutures. After double-valve replacement procedure the next step of the operation was myocardial revascularization. Arteriotomy in the medial LAD, distally of the implanted stent was performed and termino-lateral anastomosis with pedicled LIMA-graft was completed. The overall ECC time was 137 min., the aortic cross-clamp time - 99 min. and reperfusion of $34 \mathrm{~min}$.

The weaning of CPB was successfully obtained with optimal dose of catecholamines (Dopamine).

\section{POSTOPERATIVE PERIOD}

The patient was transferred into the intensive care unit, where the hemodynamic parameters of the patient deteriorated in the first hours after the operation. On the 3th postoperative hour the total bleeding was $610 \mathrm{ml}$ and revision of the patient was performed. On the second postoperative day the bleeding from the mediastinal drainages was $550 \mathrm{ml}$ and from the left chest tube $-540 \mathrm{ml}$. On the third postoperative day a second revision for acute pericardial tamponade was performed and $600 \mathrm{ml}$ of blood collection was evacuated. On the fifth postoperative day $160 \mathrm{ml}$ was drained from the retrosternal tube drainage and $610 \mathrm{ml}$ from the left chest tube, which required a biochemical analysis of the pleural exudate. The results demonstrated high triglycerides levels and deviation from the normal protein and cholesterol levels and high values of blood sugar and lactate dehydrogenase. On the sixth postoperative day, the retrosternal drain was removed. From the left chest tube $740 \mathrm{ml}$ were evacuated. Treatment with Octreotide was initiated (Sandostatin amp, Novartis Pharmaceuticals, East Hannover, NJ) 100 micrograms, subcutaneously on every eight hours and a total parenteral nutrition with a solution rich of proteins and medium chain triglycerides-OliClinomel. On the eighth postoperative day, the subcutaneous applications of Octreotide were replaced by continuous intravenous infusion. By postoperative day twelve the amount of drained fluid through the left chest tube was increasing, which required revision of the left 
pleural cavity. Until thirteenth post-operative day the amount of fluid drained from the left pleural cavity reached $13,040 \mathrm{ml}$. A left lateral thoracotomy was performed and $300 \mathrm{ml}$ of milk-like fluid was evacuated.

The lesion of the thoracic duct was visualized at the level of Th-6. A ligature of the thoracic duct with two "U"-shaped 4/0 prolene sutures in normal periductal tissue was performed. Then the suture was secured with two-component tissue glue "Tissucol". Before the replacement of the chest drainage and closure of the thoracotomy a mechanical pleurodesis across the entire surface of the left chest wall was completed. The patient was then transferred again to intensive care unit. An intravenous infusion of Sandostatin was initiated. On the second day after the revision of the left thoracic cavity the total amount of fluid drained through the chest tube reached $240 \mathrm{ml}$, then the drainage was removed and the intravenous infusion of Octreotide - discontinued. On the seventeenth postoperative day, the patient showed symptoms of oligo-anuria and laboratory tests revealed renal failure: urea $-47 \mathrm{mmol} / \mathrm{l}$, creatinine - $485 \mathrm{micromol} / \mathrm{l}$, which required continuous venovenous hemodiafiltration). The ultrasound studies revealed the presence of residual $100 \mathrm{ml}$ of left pleural effusion. The combined conservative and surgical approach to this serious complication was effective and the patient was discharged on postoperative day 32 with improvement, in good overall health and without ultrasound or X-ray evidence of left pleural effusion.

\section{DISCUSSION}

The persistent postoperative chylothorax is a serious and life-threatening condition with incidence of 0.3$1.5 \%$ after cardiac surgery. The thoracic duct and its tributaries could be affected during esophagectomy, other mediastinal exploration or congenital heart surgery operations [3]. Chylothorax also could be observed after CABG-operations due to the harvesting of the left internal thoracic artery. In our institution for the period - January 2011-2019 - postoperative chylothorax was observed in 1 of all 3156 patients who underwent CABG - operation with the use of LIMA for arterial graft.

The conventional and contemporary treatment of chylothorax includes drainage of the pleural cavity, total parenteral feeding and surgical ligation of the thoracic duct. Minimally-invasive technique like VATS, established in 1990 is preferred when possible [4]. The conventional and more aggressive surgical options are those with transthoracic or transabdominal approaches for ligation of the thoracic duct. The pleuro-peritoneal shunt also should be considered in the cases of severe chylothorax for more than 2-3 weeks and with secretion more than $1000 \mathrm{ml} / \mathrm{day}$. The surgical results are not always successful and some complications could be observed. The condition of chylothorax is often associated with critical loss of fluids, proteins, lymphocytes, factors of the coagulation and antibodies, so in these cases the conservative strategy for treatment should be considered [5].

Cerfolio et al. reported of re-operations in 34 of 47 patients, diagnosed with chylothorax. In 23 of 34 patients $(68 \%)$, during the re-operation traumatic lesion of the thoracic duct was discovered, complications were observed in 18 of them and in 3 patients another surgery was required [2].

There are a few pharmacological agents, different than octreotide, which were used for the treatment of postoperative chylothorax. OK432 - Picibanil induces chemical pleurodesis, but it was used in only 1 patient. The intravenous infusion of ethylephrine hydrochloride with its sympathomimetic effect on the smooth muscle cells in the lymph vessels reduces the lymphatic drainage. This drug was used in 10 patients and was effective in 8 of them. In the other 2 patients, reoperation was performed [6, 7].

Octreotide is a long-lasting somatostatin analogue and causes direct effect on the vascular receptors for somatostatin and reduces the secretion of lymph fluid. Also, octreotide increases the arteriolar vascular resistance in the splanchnicus, which reduces the blood flow in GIT, which also reduces the lymph drainage. Some authors mention other effect of octreotide: reduce secretion by the pancreas and the gallbladder [8].

Some researchers reported for successfully treated chylothorax after cardiac surgery in newborns and infants with the use of octreotide. There is no information in the literature for recurrent chylothorax after octreotide therapy. Some adverse effects of the therapy with octreotide are: headache, gastrointestinal reactions (cramps, nausea, vomiting and diarrhea or constipation), epistaxis. There were no adverse effects in our patient. The intravenous administration of octreotide could be used as an initial conservative step in the treatment of chylothorax.

\section{CONCLUSION}

Surgical treatment of chylothorax is indicated in adults, if the loss of lymph is more than $1000 \mathrm{ml} /$ day 
on consecutive days. The VATS - technique is the preferred technique for the operation, but in case of failure of the procedure, the conventional surgical treatment should be considered [7].

\section{REFERENCES}

1. Allen EM, Van Heeckeren DW, Spector ML, Blumer JL. Management of nutritional and infectious complications of postoperative chylothorax in children. J Pediatr Surg 1991; 26:1169-74.

2. Cerfolio RJ, Allen MS, Deschamps $C$ et al. Postoperative chylothorax. J Thorac Cardiovasc Surg 1996 ;112:1361-6.

3. Graham DD, McGahren ED, Tribble CG, Daniel TM, Rod treatment of chylothorax. Ann Thorac Surg 1994;57:1507-12.
4. Kumar S, Kumar A, Pawar DK. Thoracoscopic management of thoracic duct injury: Is there a place for conservatism? J Postgrad Med 2004;50:57-9.

5. Nakano A, Kato M, Watanabe T et al. OK-432 chemical pleurodesis for the treatment of persistent chylothorax Hepatogastroenterology 1994:41: 568-70.

6. Nguyen DM, Shum-Tim D, Dobell AR, Tchervenkov Cl. The management of chylothorax/chylopericardium following pediatric cardiac surgery: a 10-year experience. J Card Surg 1995:302-8.

7. Wurnig PN, Hollaus PH, Ohtsuka T et al. Thoracoscopic direct clipping of the thoracic duct for chylopericardium and chylothorax. Ann Thorac Surg 2000:70: 1662-5.

8. Valentine VG, Raffin TA. The management of chylothorax. Chest 1992:102:586-91.

Accepted 10.07.2019 